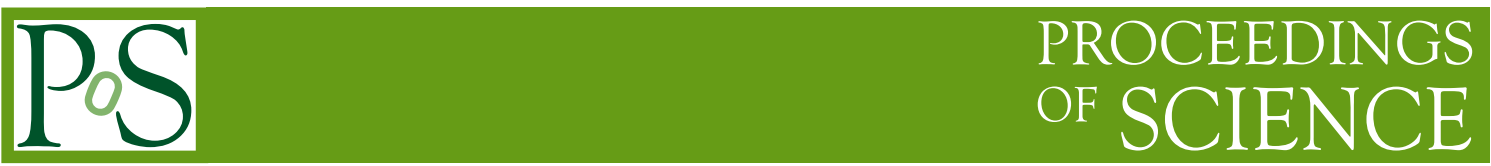

\title{
Extension of the ratio method to low energy
}

\section{F. Colomer ${ }^{* a}$, P. Capel ${ }^{a}$, F. M. Nunes ${ }^{b}$ and R. C. Johnson ${ }^{c}$}

${ }^{a}$ Physique Nucléaire et Physique Quantique CP. 229, Université Libre de Bruxelles (ULB),

B-1050 Brussels, Belgium

${ }^{b}$ National Superconducting Cyclotron Laboratory and Department of Physics and Astronomy,

Michigan State University (MSU), East Lansing, Michigan 48824, USA

${ }^{c}$ Department of Physics, University of Surrey, Guildford GU2 7XH, United Kingdom

E-mail: fcolomer@ulb.ac.be

\begin{abstract}
The ratio method is a recent observable that has been proposed for the study of halo nuclei [1]. It consists of the ratio of breakup angular distribution and the summed angular distribution (which includes elastic, inelastic and breakup) and removes the reaction model dependence. Originally, this method was developed for high and intermediate energies and studies of the reactions of ${ }^{11} \mathrm{Be}$ halo nucleus on ${ }^{12} \mathrm{C}$ and ${ }^{208} \mathrm{~Pb}$ targets at $70 \mathrm{MeV} / \mathrm{u}$ have shown this observable to provide precise information about the halo structure [2]. Given the potential interest in applying this method at lower energy, we explore its validity at beam energies of $20 \mathrm{MeV} / \mathrm{u}$ in this work. We use the Continuum Discretized Coupled Channel method and the Coulomb-corrected Dynamical Eikonal Approximation for the study of the reactions of ${ }^{11} \mathrm{Be}$ on ${ }^{12} \mathrm{C},{ }^{40} \mathrm{Ca}$ and ${ }^{208} \mathrm{~Pb}$ at $20 \mathrm{MeV} / \mathrm{u}$. We compare the various theoretical descriptions and explore the dependence of our results on the core-target interaction. Our study demonstrates that the ratio method is still valid at these lower beam energies [3]. This opens up the way to its experimental use in ISOL-type laboratories.
\end{abstract}

54th International Winter Meeting on Nuclear Physics

25-29 January 2016

Bormio, Italy

${ }^{*}$ Speaker. 


\section{Introduction}

Halo nuclei are light and very neutron-rich nuclei that can be found far from stability. First observed in the mid-80s by Tanihata et al. [4], they are characterized by a very large matter radius. While most nuclei are compact, halo nuclei can be seen as a compact and inert core surrounded by one or two very weakly bound valence neutrons. These valence neutrons form a long tail in the matter distribution, extending well beyond the range of the nuclear force. Examples of neutronhalo nuclei include ${ }^{11} \mathrm{Be}$ and ${ }^{15} \mathrm{C}$ for one-neutron halos and ${ }^{6} \mathrm{He}$ and ${ }^{11} \mathrm{Li}$ for two-neutron halos. Proton halos also exist for proton-rich nuclei, such as ${ }^{8} \mathrm{~B}$. But their halo presents a much smaller spatial extent due to the Coulomb barrier.

The small binding energies of the valence neutrons causes these nuclei to be very unstable. Their lifetimes being relatively short, usually ranging from a few milliseconds to a few seconds, these nuclei must be studied through reactions such as elastic scattering, transfer or knockout reactions. However, the most popular way to study halo nuclei is through breakup reactions, where the halo nucleus dissociates by interacting with a given target. The cross sections for this type of reactions are large and provide information about the binding energy, the angular momentum and the size of the halo system [5]. However, slight changes in the reaction model can cause significant changes in these cross sections.

To ensure that the extraction of the halo nucleus structure information is not biased by the reaction model, Capel, Johnson and Nunes proposed the ratio method in Refs.[1, 2]. It consists of the ratio of the differential breakup angular distribution on the sum of the elastic and the total breakup angular distributions. This ratio removes most of the dependence on the reaction mechanism and is then sensitive only to the projectile structure. In Refs.[1, 2], the analysis of the reaction of a ${ }^{11} \mathrm{Be}$ projectile with ${ }^{12} \mathrm{C}$ and ${ }^{208} \mathrm{~Pb}$ targets at energies of the projectile around $70 \mathrm{MeV} /$ nucleon showed the ratio to be indeed independent of the reaction mechanism and to provide information on the projectile structure in a model-independent way.

This choice of energy was motivated by the fact that many previous breakup experiments have been conducted in this energy regime. However, poor precision on the measurement of both elastic and breakup cross sections can be achieved at these energies since the process is very forwardfocused. A better precision can be achieved by considering these reactions at lower energy. In this work, we study the reliability of the ratio method and its sensitivity to the projectile structure at energies of the projectile around $20 \mathrm{MeV} /$ nucleon, achievable by facilities like SPIRAL 2 and FRIB.

\section{Theoretical framework}

As said earlier, the ${ }^{11} \mathrm{Be}$ nucleus is a one-neutron halo nucleus. Thanks to the large spatial extension of the valence neutron and to its small binding energy, it is well described by a two-body structure. The two-body projectile $P$ of mass $m_{P}$ consists of a valence neutron $n$ of mass $m_{n}$, loosely bound to a core $c$ of mass $m_{c}\left({ }^{10} \mathrm{Be}\right)$, which is assumed to be structureless and in its ground-state of spin and parity $0^{+}$. In this work, the spin of the valence neutron is neglected. The projectile is then impinging on a structureless target $T$ of mass $m_{T}$ and we thus consider a three-body model for of the collision reaction (Fig. 1). 


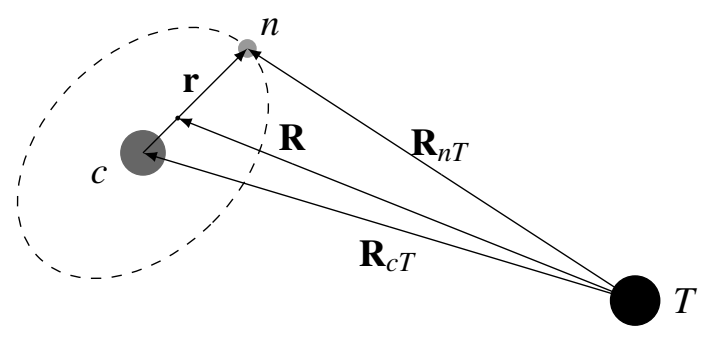

Figure 1: Jacobi set of coordinates.

To describe the $c-n$ interaction, we use the phenomenological potential $V_{c n}(\mathbf{r})$. The $c-T$ and the $n-T$ interactions are reproduced by the $V_{c T}\left(\mathbf{R}_{c T}\right)$ and $V_{n T}\left(\mathbf{R}_{n T}\right)$ potentials. In the $\{\mathbf{r}, \mathbf{R}\}$ Jacobi set of coordinates, the $c-T$ and $n-T$ coordinates read $\mathbf{R}_{c T}=\mathbf{R}-\frac{m_{n}}{m_{P}} \mathbf{r}$ and $\mathbf{R}_{n T}=\mathbf{R}+\frac{m_{c}}{m_{P}} \mathbf{r}$ respectively. $V_{c n}$ is chosen to reproduce the low-energy states of the projectile while $V_{c T}$ and $V_{n T}$ are optical potentials chosen to reproduce the elastic scattering cross section of the core and neutron with the target.

The Hamiltonian describing the projectile reads

$$
H_{0}=-\frac{\hbar^{2}}{2 \mu_{c n}} \Delta_{\mathbf{r}}+V_{c n}(\mathbf{r})
$$

where $\mu_{c n}$ is the core-neutron $c-n$ reduced mass. Its eigenstates satisfy

$$
\left(H_{0}-E\right) \phi_{l m}(E, \mathbf{r})=0,
$$

where $E$ is the $c-n$ relative energy, $l$ is the $c-n$ orbital angular momentum and $m$ its projection. The bound states of the projectile correspond to the negative energy states $E<0$ while the positiveenergy states $E>0$ describe the $c-n$ continuum and correspond to a broken-up projectile.

The three-body Schrödinger equation for the collision reads

$$
\left[-\frac{\hbar^{2}}{2 \mu_{P T}} \Delta_{\mathbf{R}}+H_{0}+V_{c T}\left(\mathbf{R}_{c T}\right)+V_{n T}\left(\mathbf{R}_{n T}\right)\right] \Psi(\mathbf{r}, \mathbf{R})=E_{\mathrm{tot}} \Psi(\mathbf{r}, \mathbf{R}),
$$

where $\mu_{P T}$ is the $P-T$ reduced mass and $\Psi$ is the three-body wave function. This equation must be solved with the condition that the projectile is impinging in the $Z$ direction with initial $P-T$ relative momentum $\hbar K_{0}$ and is initially in its ground state $\phi_{l_{0} m_{0}}$ with energy $E_{0}$, which reads

$$
\Psi(\mathbf{r}, \mathbf{R}) \underset{Z \rightarrow-\infty}{\longrightarrow} e^{i K_{0} Z} \phi_{l_{0} m_{0}}\left(E_{0}, \mathbf{r}\right) .
$$

In this work, we solve this equation with the Continuum Discretised Coupled Channel method (CDCC) [6] and within the Dynamical Eikonal Approximation corrected for Coulomb (CC-DEA) [7, 8]. In the first one, $\Psi$ is projected onto the complete set of eigenstates of the $c-n$ system. We discretise the continuum by averaging scattering states over small energy bins. The Dynamical Eikonal Approximation is based on an eikonal approximation, which assumes a sufficiently high energy to consider that the projectile trajectory does not deviate much from a straight line. The three-body wave function can hence be factorized into a plane wave times a function varying smoothly with $\mathbf{R}$, which then simplifies the equation to be solved. [7] 
However, as said earlier, breakup cross sections are highly model-dependent and the extraction of the information is flawed by uncertainties, especially the core-target interaction, mostly unknown. Indeed, as can be seen on Fig. 2, slight changes in the effective interactions $V_{c T}$ and $V_{n T}$ or even the numerical method used for the calculations can lead to significant variations of the cross sections. The same occurs when changing the target, as can be seen on Fig. 3. A different observable containing information about the halo structure and not biased by the reaction model used to calculate it is then needed: the ratio.
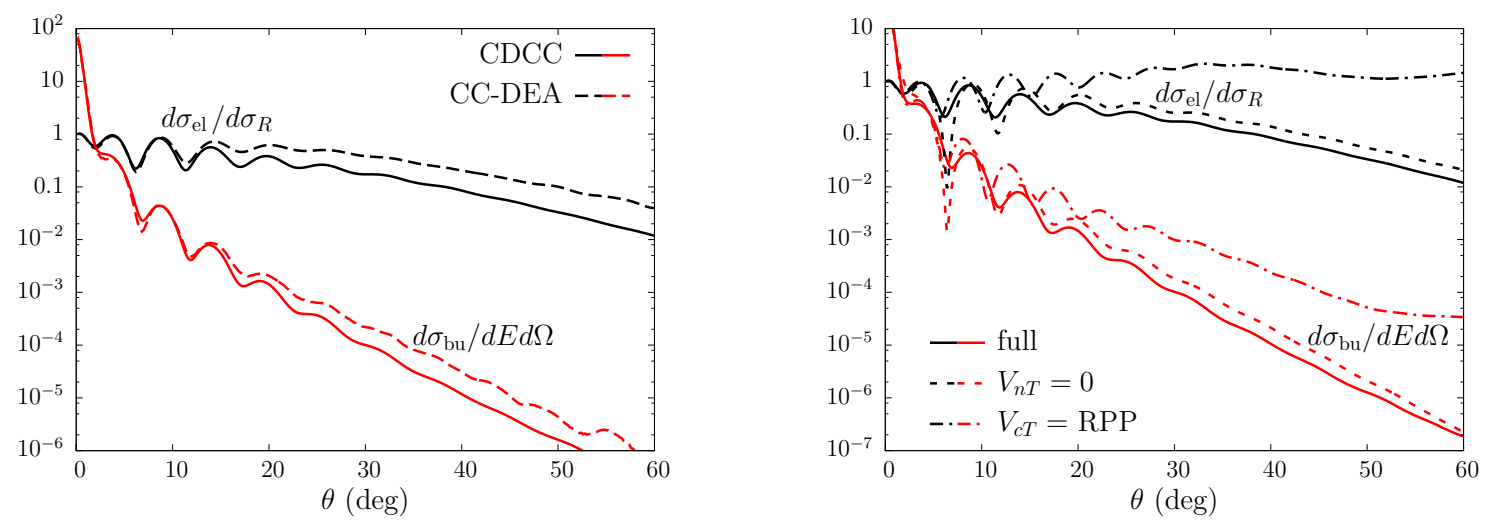

Figure 2: Differential breakup (red) and elastic scattering (black) angular distributions for the reaction of ${ }^{11} \mathrm{Be}$ on ${ }^{12} \mathrm{C}$ at $20 \mathrm{MeV} /$ nucleon. Influence of the numerical solving method (left) and the effective interactions (right). RPP potential taken from [10]
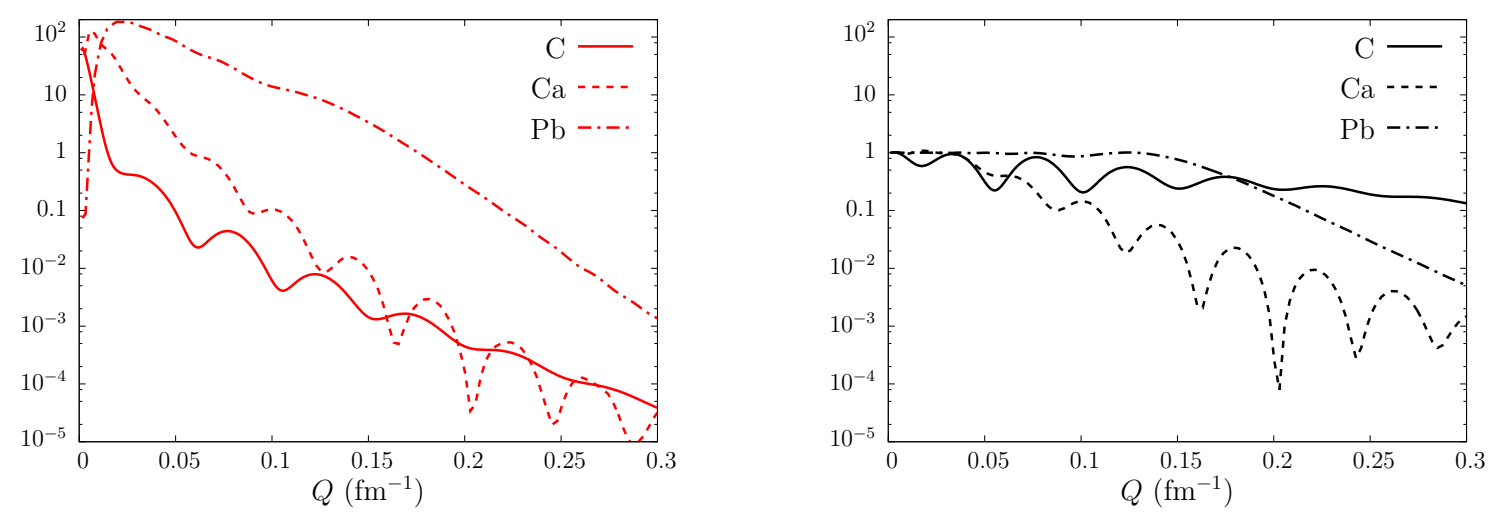

Figure 3: Differential breakup (red, left) and elastic scattering (black, right) angular distributions for the reaction of ${ }^{11} \mathrm{Be}$ on ${ }^{12} \mathrm{C},{ }^{40} \mathrm{Ca}$ and ${ }^{208} \mathrm{~Pb}$ at $20 \mathrm{MeV} /$ nucleon $(Q$ is proportional to the transferred momentum $\mathbf{Q}=m_{n} / m_{P}\left(\mathbf{K}_{0}-\mathbf{K}^{\prime}\right)$ )

\section{The Ratio}

The idea of the ratio comes primarily from the Recoil Excitation and Breakup model (REB) [9]. Let us then solve the Schrödinger equation in this model, which assumes an adiabaticor sudden-treatment of the projectile excitation $\left(H_{0} \simeq E_{0}\right)$ and neglects the $n-T$ interaction 
$\left(V_{n T}=0\right)$. With these hypotheses, the elastic-scattering cross section factorises into the product of the cross section for a pointlike projectile of mass $\mu_{P T}$ scattered by the $c-T$ interaction, times a form factor accounting for the halo structure. The factorised cross section reads

$$
\left(\frac{d \sigma}{d \Omega}\right)_{\mathrm{el}} \stackrel{(\mathrm{REB})}{=}\left|F_{0,0}\right|^{2} \times\left(\frac{d \sigma}{d \Omega}\right)_{\mathrm{pt}}
$$

where the form factor reads explicitly $F_{0,0}=\int\left|\phi_{l_{0} m_{0}}\right|^{2} e^{i \mathbf{Q} \cdot \mathbf{r}} d \mathbf{r}$ and represents the Fourier transform of the halo ground-state density (where $\mathbf{Q}=m_{n} / m_{P}\left(\mathbf{K}_{0}-\mathbf{K}^{\prime}\right.$ ) is proportional to the transferred momentum of the scattering process).

The same occurs for the cross section for breakup and for inelastic scattering. In the particular case of breakup to the energy $E>0$ in the continuum, the form factor reads

$$
\left|F_{E, 0}\right|^{2}=\sum_{l m}\left|\int \phi_{l m}(E, \mathbf{r}) \phi_{l_{0}, m_{0}} e^{i \mathbf{Q} \cdot \mathbf{r}} d \mathbf{r}\right|^{2}
$$

The main interest of the factorisation (3.1) is the fact that the not-well-known pointlike projectile cross section can thus be canceled by taking the ratio of any linear combination of these different cross sections [1,2]. We are then left with an observable consisting of the ratio of form factors, accounting for the halo structure only and hence totally independent of the reaction process. In this work, following the idea of Refs.[1, 2], we study the ratio

$$
\mathcal{R}_{\text {sum }}(E, \mathbf{Q}) \equiv \frac{d \sigma_{\mathrm{bu}}}{d \sigma_{\mathrm{sum}}}
$$

which in the REB approximation is simply $\left|F_{E, 0}\right|^{2}$ and with

$$
\left(\frac{d \sigma}{d \Omega}\right)_{\mathrm{sum}}=\left(\frac{d \sigma}{d \Omega}\right)_{\mathrm{el}}+\sum_{i>0}\left(\frac{d \sigma_{i}}{d \Omega}\right)_{\text {inel }}+\int\left(\frac{d \sigma}{d E d \Omega}\right)_{\mathrm{bu}} d E
$$

In order to illustrate the sensitivity of the form factor $\left|F_{E, 0}\right|^{2}$ to the projectile structure, let us analyse the ratio for the reaction of ${ }^{11} \mathrm{Be}$ on ${ }^{12} \mathrm{C}$ at $20 \mathrm{MeV} /$ nucleon for different halo structures. On the left-hand side of Fig. 4, we represented the form factor obtained when modifying the binding energy or the partial-wave configuration of the valence neutron and on the right-hand side, we represented the influence of the details of the radial wave function by modifying the $c-n$ interaction. As can be observed, the form factor is sensible to the halo structure both in magnitude and in shape. The rather simple expression of the form factor allows us to extract information about the structure without appealing to heavy computations such as CDCC or CC-DEA.

Unfortunately, due to the adiabatic hypothesis of the REB, we expect Eq. (3.3) to be valid only at intermediate and high energies. The ratio $\mathcal{R}_{\text {sum }}$ has been tested for the reaction of ${ }^{11} \mathrm{Be}$ on ${ }^{12} \mathrm{C}$ and ${ }^{208} \mathrm{~Pb}$ at $70 \mathrm{MeV} /$ nucleon in Refs.[1, 2]. It has been shown to be very close to the REB prediction. However, as previously stated, poor precision on the measurement of both elastic and breakup cross sections can be achieved at these energies and a lower energy beam would be better suited for the study of the ratio. In this work, we lower the energy of the reaction and we explore the reliability of the ratio beyond the adiabatic hypothesis of the REB. 

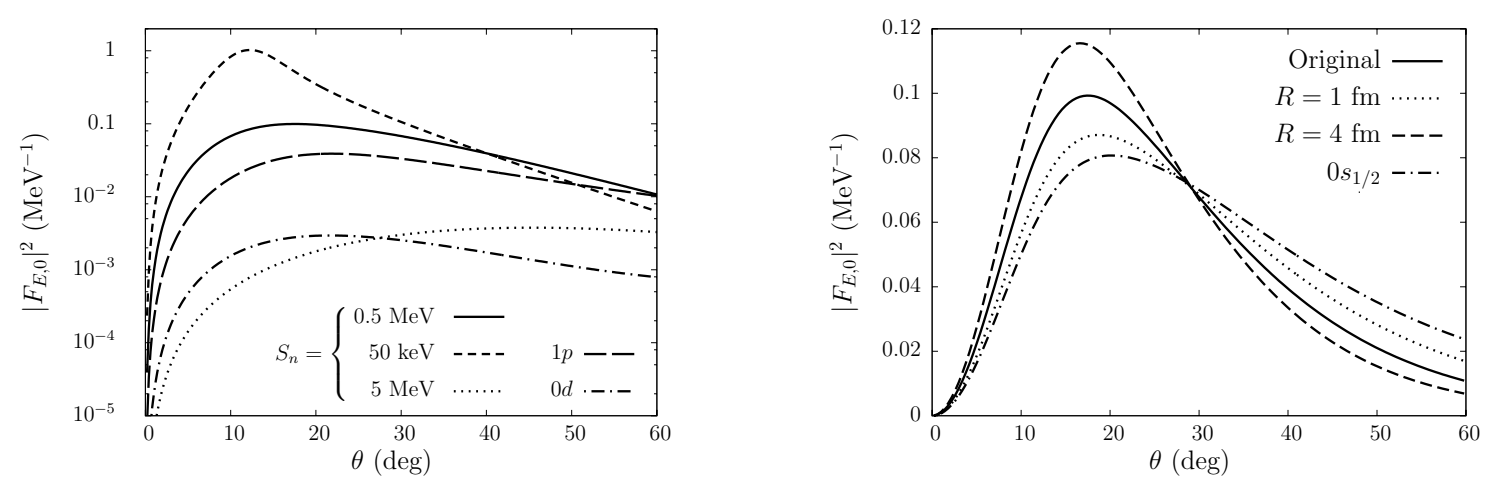

Figure 4: Sensitivity of the form factor $\left|F_{E, 0}\right|^{2}$ to the projectile structure for the reaction of ${ }^{11} \mathrm{Be}$ on ${ }^{12} \mathrm{C}$ at $20 \mathrm{MeV} /$ nucleon. On the left, influence of the binding energy and the partial-wave configuration. On the right, influence of $V_{c n}$ and hence of details in the radial wave function. Reprinted figures with permission from [3] Copyright (2016) by the American Physical Society.

The results of the calculations of the ratio at an energy of $20 \mathrm{MeV} /$ nucleon are shown on Fig. 5, for different reaction models on a ${ }^{12} \mathrm{C}$ target of the reaction on the left-hand side and for different targets on the right-hand side.

On the left-hand side, as previously observed, the slight changes in the interaction model have a significant impact on the summed and breakup cross sections (see Figs 2 and 3). However, as it had been observed in Refs.[1, 2], we remove the model dependency by taking the ratio of these cross sections. Moreover, although we are beyond the adiabatic hypothesis, $\mathcal{R}_{\text {sum }}$ and its REB prediction $\left|F_{E, 0}\right|^{2}$ are very close. Remnant oscillations still exist but they are highly attenuated and the curves are nearly superimposed, indicating that information about the projectile structure can still be reliably extracted from the ratio even at this low energy. A similar conclusion can be drawn concerning the oscillations of the ratio on the right-hand side. Moreover, the ratio $\mathcal{R}_{\text {sum }}$ removes most of the dependence on the reaction mechanism. The only significant discrepancies are found at very forward angles, where the ratio for the lead target shows a slower rise than the REB prediction. This is most certainly due to the adiabatic hypothesis, which is even less valid in the case of Coulomb-dominated processes. Indeed, the infinite-range Coulomb interaction causes the REB prediction to be overestimated. We plan to consider non-adiabatic corrections to the REB such as the one discussed in Ref.[11] and which could improve the REB prediction in the case of heavy targets at low energies.

\section{Conclusion}

We described the ratio observable, which is a recent tool for the study of halo nuclei. Previous studies at intermediate energies have shown this observable to be independent of both the mechanism and the model while still providing useful information about the halo structure, such as the binding energy or the orbital angular momentum of the valence neutron $[1,2]$. But at these energies, the experimental precision is low. In this work, we explore the reliability of the ratio at low 

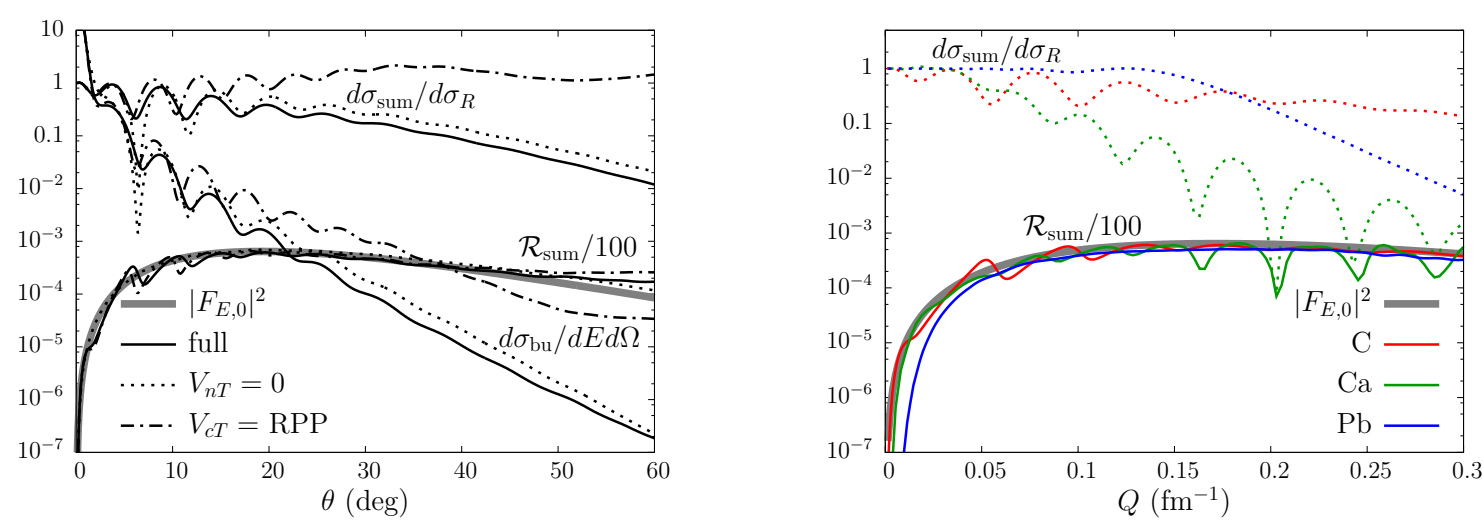

Figure 5: Independence of the reaction model (left) and target choice (right) for the ratio $\mathcal{R}_{\text {sum }}$ and comparison with its REB approximation $\left|F_{E, 0}\right|^{2}$ for the reaction of ${ }^{11} \mathrm{Be}$ on a ${ }^{12} \mathrm{C}$ target (left) and on ${ }^{12} \mathrm{C},{ }^{40} \mathrm{Ca}$ and ${ }^{208} \mathrm{~Pb}$ targets (right) at $20 \mathrm{MeV} /$ nucleon. Reprinted figure with permission from [3] Copyright (2016) by the American Physical Society.

energies, achievable by facilities like SPIRAL 2 and FRIB. We use CDCC and CC-DEA models to evaluate the ratio and we compare it to its REB prediction.

Surprisingly, the ratio and its REB prediction still agree very well. While the breakup cross sections are larger for heavy targets, the ratio seems to work better for light targets, as the infiniterange Coulomb interaction causes the REB prediction to be overestimated at very-forward angles. However, non-adiabatic corrections to the REB could solve this problem. The ratio remains modelindependent and is still sensible to the binding energy and to the orbital angular momentum. Even the discrepancies between CC-DEA and CDCC disappear when considering the ratio, suggesting that state-of-the-art reaction model and computationally-heavy calculations are not needed to extract the information from experimental measures.

This work opens the ratio method to a larger number of facilities, particularly those with lower beam energies, and will motivate groups to collect data for an experimental test of the method.

\section{Acknowledgements}

This work was supported by the National Science Foundation under Grant No. PHY-1403906, by the Department of Energy, Office of Science, Office of Nuclear Physics under Grant No. DEFG52-08NA28552, and by the Research Credit No. 19526092 of the Belgian Funds for Scientific Research F.R.S.-FNRS. This text presents research results of the Belgian Research Initiative on eXotic nuclei (BriX), Program No. P7/12 on interuniversity attraction poles of the Belgian Federal Science Policy Office. R.C.J. is supported by the UK STFC through Grant No. ST/F012012/1. This project has received funding from the European Union's Horizon 2020 research and innovation programme under grant agreement No 654002.

\section{References}

[1] P. Capel, R. C. Johnson, and F. M. Nunes, Phys. Lett. B705, 112 (2011). 
[2] P. Capel, R. C. Johnson, and F. M. Nunes, Phys. Rev. C 88, 044602 (2013).

[3] F. Colomer, P. Capel, F. M. Nunes, and R. C. Johnson, Phys. Rev. C 93, 054621 (2016).

[4] I. Tanihata et al., Phys. Rev. Lett. 55, 2676 (1985).

[5] P. Capel and F. M. Nunes, Phys. Rev. C 73, 014615 (2006).

[6] N. Austern, Y. Iseri, M. Kamimura, M. Kawai, G. Rawitscher, and M. Yahiro, Phys. Rep. 154, 125 (1987)

[7] D. Baye, P. Capel, and G. Goldstein, Phys. Rev. Lett. 95, 082502 (2005)

[8] T. Fukui, K. Ogata, and P. Capel, Phys. Rev. C 90, 034617 (2014)

[9] R. C. Johnson, J. S. Al-Khalili, and J. A. Tostevin, Phys. Rev. Lett. 79, 2771 (1997)

[10] C. M. Perey and F. G. Perey, At. Data Nucl. Data Tables 17, 1 (1976)

[11] N. C. Summers, J. S. Al-Khalili, and R. C. Johnson, Phys. Rev. C 66, 014614 (2002) 